\title{
Approximately Semigroups and Ideals: An Algebraic View of Digital Images
}

\author{
Ebubekir Inan \\ AdıyamanUniversity, Faculty of Arts and Sciences, Department of Mathematics, 3-34, 02040, Adıyaman, Turkey \\ e-posta:einan@adiyaman.edu.tr \\ This article dedicated to honor of July 15 martyrs in Turkey.
}

Geliş Tarihi: 22.12.2016 ; Kabul Tarihi: 09.08.2017

\begin{tabular}{cl} 
Anahtar kelimeler & Özet \\
\cline { 2 - 2 } $\begin{array}{c}\text { Proksimiti uzaylar; } \\
\text { Relator uzaylar; }\end{array}$ & Bu makalede proksimal relator uzaylarında yaklaşımlı yarıgruplar ve ideallere giriş yapılmıştır. Tanımsal \\
Tanımsal yaklaşımlar; & $\begin{array}{l}\text { verilmiştir. Bundan başka, nesne tanımlaması homomorfizması kullanılarak tanımsal yaklaşımların bazı } \\
\text { Yaklaşımlı yarıgruplar. }\end{array}$ \\
\cline { 2 - 2 }
\end{tabular}

\section{Yaklaşımlı Yarıgruplar ve İdealler: Dijital Görüntülerin Cebirsel incelenmesi}

\author{
Keywords \\ Proximity spaces; \\ Relator spaces; \\ Descriptive \\ approximations; \\ Approximately \\ semigroups.
}

\begin{abstract}
In this article, approximately semigroups and ideals in proximal relator spaces have beenintroduced. In addition to, some examples of approximately semigroups and ideals in digital images endowed with descriptive proximity relation have been given. Furthermore, some properties of descriptively approximations using object descriptive homomorphism have been obtained.
\end{abstract}

\section{Introduction}

The concept of ordinary algebraic structures are consist of a nonempty set of abstract points with one or more binary operations which are required to satisfy certain axioms such as a groupoid is an algebraic structure $(A, \circ)$ consist of a nonempty set $A$ and a binary operation "o" defined on $A$ (Clifford and Preston,1964). And binary operation "o" must be closed in $A$ whereas in proximal relator spaces, the sets are composed of non-abstract points instead of abstract points and these points are describable with feature vectors in. Descriptively upper approximation of a nonempty set is obtained by using the set of points composed by the proximal relator space together with matching features of points and these are the basic tools for defining algebraic structures on proximal relator spaces and binary operations on any groupoid $A$ in proximal relator space must be closed in descriptively upper approximation of $A$.

Moreover an example of approximately semigroup on digital images endowed with descriptive proximity relation has given.

\section{Preliminaries}

Let $X$ be a nonempty set. Family of relations $\mathcal{R}$ on a nonempty set $X$ is called a relator. The pair $(X, \mathcal{R})$ (or $X(\mathcal{R})$ ) is a relator space which is natural generalisations of uniform spaces (Szaz, 1987). If we consider a family of proximity relations on $X$, we have a proximal relator space $\left(X, \mathcal{R}_{\delta}\right)\left(X\left(\mathcal{R}_{\delta}\right)\right)$. As in (Peters, 2016), $\left(\mathcal{R}_{\delta}\right)$ contains proximity relations namely, Efremovič proximity $\delta$ (Efremovič, 1951;1952), Lodato proximity (Lodato, 
1962), Wallman proximity, descriptive proximity $\delta_{\Phi}$ in defining $\mathcal{R}_{\delta_{\Phi}}$ (Peters, 2013; Peters et al. 2014).

In this article, we consider the Efremovic proximity $\delta$ (Efremovič, 1952) and the descriptive proximity $\delta_{\Phi}$ in defining a descriptive proximal relator space (denoted by $\left(X, \mathcal{R}_{\delta_{\Phi}}\right)$ ).

An Efremovič proximity $\delta$ is a relation on $2^{X}$ that satisfies

1. $A \delta B \Rightarrow B \delta A$.

2. $A \delta B \Rightarrow A \neq \emptyset$ and $B \neq \emptyset$.

3. $A \cap B \neq \emptyset \Rightarrow A \delta B$.

4. $A \delta(B \cup C) \Leftrightarrow A \delta B$ or $A \delta C$.

5. $\{x\} \delta\{y\} \Leftrightarrow x=y$.

6. EF axiom. $A \underline{\delta} B \Rightarrow \exists E \subseteq X$ such that $A \underline{\delta} E$ and $E^{c} \underline{\delta} B$.

Lodato proximity (Lodato, 1962) swaps the EF axiom 2. for the following condition:

$A \delta B$ and $\forall b \in B$,

$\{b\} \delta C \Rightarrow A \delta C$. (Lodato Axiom)

In a discrete space, a non-abstract point has a location and features that can be measured (Efremovič, 1952; Kovăr, 2011). Let $X$ be a nonempty set of non-abstract points in a proximal relator space $\left(X, \mathcal{R}_{\delta_{\Phi}}\right)$ and let $\Phi=\left\{\phi_{1}, \ldots, \phi_{n}\right\}$ a set of probe functions where aprobe function $\Phi: X \rightarrow \mathbb{R}$ represents a feature of a sample point in a picture. Let $\Phi(x)=\left(\phi_{1}(x), \ldots, \phi_{n}(x)\right), n \in N$ be an object description denote a feature vector for $x$, which provides a description of each $x \in X$. To obtain a descriptive proximity relation (denoted by $\delta_{\Phi}$ ), one first choose a set of probe functions.

Definition 2.1.(Set Description, Naimpally and Peters, 2013) Let $X$ be a nonempty set of nonabstract points, $\Phi$ an object description and $A$ a subset of $X$. Then the set description of $A$ is defined as

$$
\mathcal{Q}(A)=\{\Phi(a): a \in A\}
$$

Definition 2.2.(Descriptive Set Intersection, Naimpally and Peters, 2013; Peters and Naimpally, 2012) Let $X$ be a nonempty set of non-abstract points, $A$ and $B$ any two subsets of $X$. Then the descriptive (set) intersection of $A$ and $B$ is defined as

$$
\begin{gathered}
A \underset{\Phi}{\cap} B=\{x \in A \cup B: \Phi(x) \in \mathcal{Q}(A) \text { and } \Phi(x) \\
\in \mathcal{Q}(B)\} .
\end{gathered}
$$

Definition 2.3.(Peters, 2013) Let $X$ be a nonempty set of non-abstract points, $A$ and $B$ any two subsets of $X$. If $\mathcal{Q}(A) \cap \mathcal{Q}(B) \neq \varnothing$, then $A$ is called descriptively near $B$ and denoted by $A \delta_{\Phi} B$. If $\mathcal{Q}(A) \cap \mathcal{Q}(B)=\emptyset$ then $A \underline{\delta}_{\Phi} B$ read as $A$ is descriptively far from $B$.

Definition 2.4.(Descriptive Nearness Collections, Peters, 2013) Let $X$ be a nonempty set of nonabstract points and $A$ any subset of $X$. Then the descriptive nearness collection $\xi_{\Phi}(A)$ is defined by

$$
\xi_{\Phi}(A)=\left\{B \in \mathcal{P}(X): A \delta_{\Phi} B\right\} .
$$

(Peters, et al. 2015) Let $\left(X, \mathcal{R}_{\delta_{\Phi}}\right)$ be descriptive proximal relator space and $A \subset X$, where $A$ is consist of non-abstract objects. And let $(A, \cdot)$ and $(\mathcal{Q}(A), \circ)$ be groupoids. Let consider the object description $\Phi$ by means of a function

$$
\begin{gathered}
\Phi: A \subset X \rightarrow \mathcal{Q}(A) \subset \mathbb{R}, \\
a \mapsto \Phi(a), a \in A .
\end{gathered}
$$

The object description $\Phi$ of $A \operatorname{in} \mathcal{Q}(A)$ is an object description homomorphism if

$$
\Phi(a \cdot b)=\Phi(a) \circ \Phi(b) \text { for all } a, b \in A
$$

Moreover descriptive closure of a point $a \in A$ is defined by

$$
c l_{\Phi}(a)=\{x \in X: \Phi(a)=\Phi(x)\} .
$$

Descriptively lower approximation of the set $A$ is consist of $a \in A$ which descrition of descriptive closure $\mathcal{Q}\left(c l_{\Phi}(a)\right)$ are subsets of set descriptionQ $(A)$. This discovery process leads to the construction of what is known as the descriptively lower approximation of $A \subseteq X$, which is denoted by $\Phi_{*} A$. 


\section{Main Results}

Definition 3.1. (Descriptively Lower Approximation of a Set) Let $\left(X, \mathcal{R}_{\delta_{\Phi}}\right)$ be descriptive proximal relator space and $A \subset X$. A descriptively lower approximation of $A$ is defined as

$$
\Phi_{*} A=\left\{a \in A: \mathcal{Q}\left(c l_{\Phi}(a)\right) \subseteq \mathcal{Q}(A)\right\}
$$

Definition 3.2.(Descriptively Upper Approximation of a Set) Let $\left(X, \mathcal{R}_{\delta_{\Phi}}\right)$ be descriptive proximal relator space and $A \subset X$. A descriptively upper approximation of $A$ is defined as

$$
\Phi^{*} A=\left\{x \in X: x \delta_{\Phi} A\right\}
$$

Definition 3.3.(Descriptively Boundary Region) Let $B n d_{\Phi} A$ denote the descriptively boundary region of a set $A \subseteq X$ defined by

$\Phi_{B n d} A=\Phi^{*} A \backslash \Phi_{*} A=\left\{x: x \in \Phi^{*} A\right.$ and $\left.x \notin \Phi_{*} A\right\}$.

Lemma 3.4.Let $\left(X, \mathcal{R}_{\delta_{\Phi}}\right)$ be descriptive proximal relator space and $A, B \subset X$, then

(i) $\mathcal{Q}(A \cap B)=\mathcal{Q}(A) \cap \mathcal{Q}(B)$,

(ii) $\mathcal{Q}(A \cup B)=\mathcal{Q}(A) \cup \mathcal{Q}(B)$.

Proof. (i)

$$
\begin{gathered}
\mathcal{Q}(A \cap B)=\{\Phi(x): x \in A \cap B\} \\
=\{\Phi(x): x \in A\} \cap\{\Phi(x): x \in A\} \\
=\mathcal{Q}(A) \cap \mathcal{Q}(B)
\end{gathered}
$$

(ii)

$$
\begin{gathered}
\mathcal{Q}(A \cup B)=\{\Phi(x): x \in A \cup B\} \\
=\{\Phi(x): x \in A\} \cup\{\Phi(x): x \in A\} \\
=\mathcal{Q}(A) \cup \mathcal{Q}(B)
\end{gathered}
$$

Theorem 3.5.Let $\left(X, \mathcal{R}_{\delta_{\Phi}}\right)$ be descriptive proximal relator space and $A, B \subset X$. Then the following statements hold.

(1) $\left(\Phi_{*} A\right) \subseteq A \subseteq\left(\Phi^{*} A\right)$,

(2) $\Phi^{*}(A \cup B)=\left(\Phi^{*} A\right) \cup\left(\Phi^{*} B\right)$,

(3) $\Phi_{*}(A \cap B)=\left(\Phi_{*} A\right) \cap\left(\Phi_{*} B\right)$,
(4) If $A \subseteq B$, then $\left(\Phi_{*} A\right) \subseteq\left(\Phi_{*} B\right)$,

(5) If $A \subseteq B$, then $\left(\Phi^{*} A\right) \subseteq\left(\Phi^{*} B\right)$,

(6) $\Phi_{*}(A \cup B) \supseteq\left(\Phi_{*} A\right) \cup\left(\Phi_{*} B\right)$,

(7) $\Phi^{*}(A \cap B) \subseteq\left(\Phi^{*} A\right) \cap\left(\Phi^{*} B\right)$.

Proof. (1) Let $a \in \Phi_{*} A$, then $\mathcal{Q}\left(c l_{\Phi}(a)\right) \subseteq \mathcal{Q}(A)$, where $a \in A$. Hence $\left(\Phi_{*} A\right) \subseteq A$. Let $a \in A$ and it is obvious that $\Phi(a) \in \mathcal{Q}(A)$, that is $\Phi(a) \cap \mathcal{Q}(A) \neq$ $\emptyset$ and so $a \delta_{\Phi} A$. Therefore $\mathrm{a} \in \Phi^{*} A$ and then $\mathrm{A} \subseteq \Phi^{*} A$.

By Lemma 3.4. proofs of (2) and (3) are obvious.

(4) Let $A \subseteq B$, then $A \cap B=A$. From statement (3) we have $\Phi_{*} A=\Phi_{*}(A \cap B)=\left(\Phi_{*} A\right) \cap\left(\Phi_{*} B\right)$. Hence $\left(\Phi_{*} A\right) \subseteq\left(\Phi_{*} B\right)$.

(5) Let $A \subseteq B$, then $A \cup B=B$. From statement (2) we get $\Phi^{*} B=\Phi^{*}(A \cup B)=\left(\Phi^{*} A\right) \cup\left(\Phi^{*} B\right)$. This implies that $\left(\Phi^{*} A\right) \subseteq\left(\Phi^{*} B\right)$.

(6) Since $A \subseteq A \cup B$ and $B \subseteq A \cup B$, by (4) we have $\left(\Phi_{*} A\right) \subseteq \Phi_{*}(A \cup B)$ and $\left(\Phi_{*} B\right) \subseteq \Phi_{*}(A \cup B)$. Hence $\left(\Phi_{*} A\right) \cup\left(\Phi_{*} B\right) \subseteq \Phi_{*}(A \cup B)$.

(7) We know that $A \cap B \subseteq A$ and $A \cap B \subseteq B$. From statement (5) we have $\Phi^{*}(A \cap B) \subseteq\left(\Phi^{*} A\right)$ and $\Phi^{*}(A \cap B) \subseteq\left(\Phi^{*} B\right)$. Thus $\Phi^{*}(A \cap B) \subseteq\left(\Phi^{*} A\right) \cap$ $\left(\Phi^{*} B\right)$.

Definition 3.6.Let $\left(X, \mathcal{R}_{\delta_{\Phi}}\right)$ be descriptive proximal relator space and let "." be binary operation defined on $X$. A subset $G$ of the set of $X$ is called $a$ descriptive approximately groupoid in descriptive proximal relator space if $x \cdot y \in \Phi^{*} G, \forall x, y \in G$.

Suppose that $G$ is a descriptive approximately groupoid with the binary operation "." in $\left(X, \mathcal{R}_{\delta_{\Phi}}\right)$, $g \in G$ and $A, B \subseteq G$. We define the subsets $g \cdot A, A \cdot g, A \cdot B \subseteq \Phi^{*} G \subseteq X$ as follows:

$$
\begin{aligned}
& g \cdot A=g A=\{g a: a \in A\}, \\
& A \cdot g=A g=\{a g: a \in A\},
\end{aligned}
$$

$A \cdot B=A B=\{a b: a \in A, b \in B\}$. 
Lemma 3.7.Let $\left(X, \delta_{\Phi}\right)$ be descriptive proximity space and $A, B \subset X$. If $\Phi: X \rightarrow \mathbb{R}$ is an object descriptive homomorphism, then

Proof.

$$
\mathcal{Q}(A) \mathcal{Q}(B)=\mathcal{Q}(A B)
$$

$$
\mathcal{Q}(A) \mathcal{Q}(B)
$$$$
\begin{aligned}
& =\{\Phi(a) \Phi(b): a \in A, b \in B\} \\
= & \{\Phi(a b): a \in A, b \in B\}=Q(A B)
\end{aligned}
$$

Theorem 3.8.Let $\left(X, \delta_{\Phi}\right)$ be descriptive proximity space and $A, B \subset X$. If $\Phi: X \rightarrow \mathbb{R}$ is an object descriptive homomorphism, then

$$
\left(\Phi^{*} A\right)\left(\Phi^{*} B\right)=\Phi^{*}(A B) .
$$

Proof. Suppose that $x \in\left(\Phi^{*} A\right)\left(\Phi^{*} B\right)$, then $x=a b$ with $a \in \Phi^{*} A$ and $b \in \Phi^{*} B$. Thus $\Phi(a) \in \mathcal{Q}(A)$ and $\Phi(b) \in \mathcal{Q}(B)$. Since $\Phi$ is an object descriptive homomorphism $\Phi(a) \Phi(b)=\Phi(a b) \in \mathcal{Q}(A) \mathcal{Q}(B)$ and so by Lemma 3.7. $\Phi(x) \in Q(A B)$, that is $x \in \Phi^{*}(A B)$. Therefore $\left(\Phi^{*} A\right)\left(\Phi^{*} B\right) \subset \Phi^{*}(A B)$. Similary we obtain $\Phi^{*}(A B) \subset\left(\Phi^{*} A\right)\left(\Phi^{*} B\right)$ and so $\left(\Phi^{*} A\right)\left(\Phi^{*} B\right)=\Phi^{*}(A B)$.

Theorem 3.9Let $\left(X, \delta_{\Phi}\right)$ be descriptive proximity space, $A, B \subset X$. If $\Phi: X \rightarrow \mathbb{R}$ is an object descriptive homomorphism, then

$$
\left(\Phi_{*} A\right)\left(\Phi_{*} B\right) \subset \Phi_{*}(A B) .
$$

Proof. Let $x \in\left(\Phi_{*} A\right)\left(\Phi_{*} B\right)$, then $x=a b$ with $a \in \Phi_{*} A$ and $b \in \Phi_{*} B$. Thus $\mathcal{Q}\left(\operatorname{cl}_{\Phi}(a)\right) \subseteq \mathcal{Q}(A)$, $\mathcal{Q}\left(c l_{\Phi}(b)\right) \subseteq \mathcal{Q}(B)$ and so $y \in X$ where $\Phi(a)=\Phi(y) \in \mathcal{Q}(A), \quad \Phi(b)=\Phi(y) \in \mathcal{Q}(B)$. Since $\Phi$ is an object descriptive homomorphism $\Phi(a) \Phi(b)=\Phi(a b)=\Phi(y) \in \mathcal{Q}(A) \mathcal{Q}(B) \quad$ and from Lemma 3.7. $\Phi(a b)=\Phi(y) \in Q(A B)$. Then $\mathcal{Q}\left(c l_{\Phi}(a b)\right) \subseteq \mathcal{Q}(A B)$, that is $x=a b \in \Phi_{*}(A B)$. Consequently $\left(\Phi_{*} A\right)\left(\Phi_{*} B\right) \subset \Phi_{*}(A B)$.

\subsection{Approximately Semigroups and Ideals}

Definition 3.10. Let $\left(X, \mathcal{R}_{\delta_{\Phi}}\right)$ be descriptive proximal relator space and "." be a binary operation defined on $X . S \subset X$ is called an approximately semigroup in descriptive proximal relator space if the following properties are verified:
1. $x \cdot y \in \Phi^{*} S, \forall x, y \in S$.

2. $(x \cdot y) \cdot z=x \cdot(y \cdot z)$ property verify on $\Phi^{*} S, \forall x, y, z \in S$.

Let an approximately semigroup has approximately identity element $e \in \Phi^{*} S$ such that $x \cdot e=e \cdot x=$ $(A B)$ $x, \forall x \in S$. Then $S$ is called an approximately monoid in a descriptive proximal relator space.

Let $x \cdot y=y \cdot x, \forall x, y \in S$ property holds in $\Phi^{*} S$. Then $S$ is commutative approximately semigroup in descriptive proximal relator space.

Example 3.11.Let $X$ be a digital image endowed with descriptive proximity relation $\delta_{\Phi}$ and consists of 25 pixels as in Fig. 1.

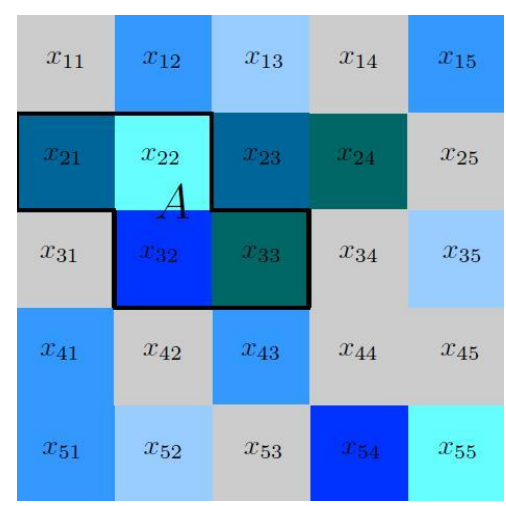

Figure 1: Digital image

A pixel $x_{i j}$ is an element at position $(i, j)$ (row and column) in digital image $X$. Let $\phi$ be a probe function that represent RGB colour of each pixel are given in Table 1.

Table 1. RGB codes of each pixel in Fig. 1.

\begin{tabular}{ccccccccc} 
& Red & Green & Blue & & & Red & Green & Blue \\
\cline { 1 - 1 }$x_{11}$ & 204 & 204 & 204 & & $x_{34}$ & 204 & 204 & 204 \\
$x_{12}$ & 51 & 153 & 255 & & $x_{35}$ & 204 & 255 & 255 \\
$x_{13}$ & 204 & 255 & 255 & & $x_{41}$ & 51 & 153 & 255 \\
$x_{14}$ & 204 & 204 & 204 & & $x_{42}$ & 204 & 204 & 204 \\
$x_{15}$ & 51 & 153 & 255 & & $x_{43}$ & 51 & 153 & 255 \\
$x_{21}$ & 0 & 102 & 153 & & $x_{44}$ & 204 & 204 & 204 \\
$x_{22}$ & 102 & 255 & 255 & $x_{45}$ & 204 & 204 & 204
\end{tabular}




$\begin{array}{lcccccc}x_{23} & 0 & 102 & 153 & x_{51} & 51 & 153 \\ x_{24} & 0 & 102 & 102 & x_{52} & 204 & 255 \\ x_{25} & 204 & 204 & 204 & x_{53} & 204 & 204 \\ x_{31} & 204 & 204 & 204 & x_{54} & 0 & 51 \\ x_{32} & 0 & 51 & 255 & x_{55} & 102 & 255 \\ x_{33} & 0 & 102 & 102 & & & \end{array}$

Let

$$
\begin{gathered}
\cdot: X \times X \rightarrow X \\
\left(x_{i j}, x_{k l}\right) \mapsto x_{i j} \cdot x_{k l}=x_{p r}
\end{gathered}
$$

where $p=\min \{i, k\}$ and $r=\min \{j, l\}$

be a binary operation on $X$ and $A=\left\{x_{21}, x_{22}, x_{32}, x_{33}\right\}$ be a subimage (subset) of $X$.

We can compute the descriptively upper approximation of $A$ by using the Definition 3.2. $\Phi^{*} A=\left\{x_{i j} \in X: \delta_{\phi} A\right\}, \quad$ where $\quad \mathcal{Q}(A)=$ $\left\{\phi\left(x_{i j}\right): x_{i j} \in A\right\}$. Then $\phi\left(x_{i j}\right) \cap \mathcal{Q}(A) \neq \varnothing$ such that $x_{i j} \in X$. From Table 1, we obtain

$$
\begin{gathered}
\mathcal{Q}(A) \quad=\left\{\phi\left(x_{21}\right), \phi\left(x_{22}\right), \phi\left(x_{32}\right), \phi\left(x_{33}\right)\right\} \\
=\{(0,102,153),(102,255,255), \\
(0,51,255),(0,102,102)\}
\end{gathered}
$$

Hence we get

$$
\Phi^{*} A=\left\{x_{21}, x_{22}, x_{23}, x_{24}, x_{32}, x_{33}, x_{54}, x_{55}\right\}
$$

as shown in Fig. 2.

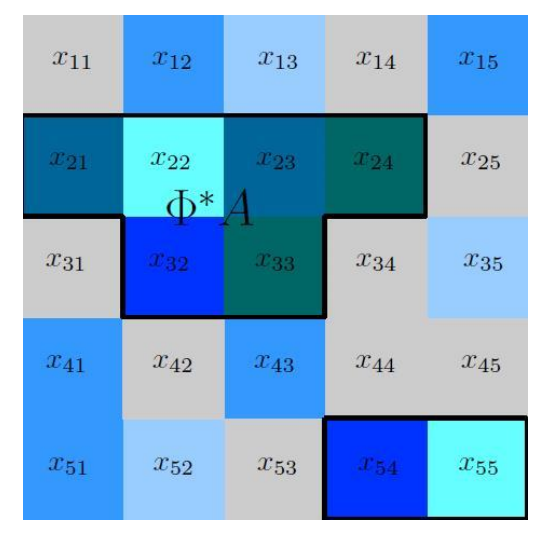

Figure 2: $\Phi^{*} A$
Since

1. For all $x_{i j}, x_{k l} \in A, x_{i j} \cdot x_{k l} \in \Phi^{*} A$,

2. For all $x_{i j}, x_{k l}, x_{m n} \in A,\left(x_{i j} \cdot x_{k l}\right) \cdot x_{m n}=$ $x_{i j} \cdot\left(x_{k l} \cdot x_{m n}\right)$ property holds in $\Phi^{*} A$,

are satisfied, the subimage $A$ of the digital image $X$ is indeed an approximately semigroup in descriptive proximity space $\left(X, \delta_{\Phi}\right)$ with binary operation ". ". Moreover, since $x_{i j} \cdot x_{k l}=x_{k l}$. $x_{i j}$, for all $x_{i j}, x_{k l} \in A$ property holds in $\Phi^{*} A, A$ is a commutative approximately semigroup.

Notice that in Example 3.11 proximal identity element is not unique. $x_{33}$ and $x_{55} \in \Phi^{*} A$ have feature ofa proximal identity element. So Adoes nothave an unique identity element and $A$ is not a commutative approximately monoid.

Example 3.12. Let $X$ be a digital image endowed with descriptive proximity relation $\delta_{\Phi}$ and consists of 16 pixels as in Fig. 3.

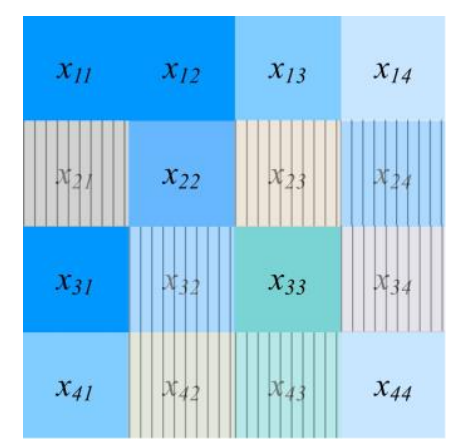

Figure 3: Digital image $X$ and $S \subset X$

\begin{tabular}{|c|c|c|c|c|c|c|c|}
\hline & Red & Green & Blue & & Red & Green & Blue \\
\hline$x_{11}$ & 0 & 151 & 255 & $x_{31}$ & 0 & 151 & 255 \\
\hline$x_{12}$ & 0 & 151 & 255 & $x_{32}$ & 103 & 183 & 255 \\
\hline$x_{13}$ & 130 & 255 & 255 & $x_{33}$ & 121 & 212 & 211 \\
\hline
\end{tabular}

A pixel $x_{i j}$ is an element at position $(i, j)$ (row and column) in $X$. Let $\phi$ be a probe function that represents RGB colour of each pixel are given in Table 2.

Table 2. RGB codes of each pixel in Fig. 3. 


$\begin{array}{llllllll}x_{14} & 200 & 204 & 255 & x_{34} & 205 & 205 & 216 \\ x_{21} & 170 & 153 & 170 & x_{41} & 130 & 205 & 255 \\ x_{22} & 103 & 102 & 255 & x_{42} & 202 & 210 & 187 \\ x_{23} & 224 & 255 & 187 & x_{43} & 121 & 212 & 211 \\ x_{24} & 103 & 102 & 255 & x_{44} & 200 & 230 & 255\end{array}$

Let

$$
\begin{gathered}
\because X \times X \rightarrow X \\
\left(x_{i j}, x_{k l}\right) \mapsto x_{i j} \cdot x_{k l}=x_{p r}, \\
p=\min \{i, k\} \text { and } r=\min \{j, l\}
\end{gathered}
$$

be a binary operation on $X$ and

$$
S=\left\{x_{21}, x_{23}, x_{24}, x_{32}, x_{34}, x_{42}, x_{43}\right\}
$$

a subimage (subset) of $X$.

We can compute the descriptively upper approximation of $S$ by using the Definition 3.2. $\Phi^{*} S=\left\{x_{i j} \in X: x_{i j} \delta_{\phi} S\right\}, \quad$ where $\mathcal{Q}(S)=$ $\left\{\phi\left(x_{i j}\right): x_{i j} \in S\right\}$. Then $\phi\left(x_{i j}\right) \cap \mathcal{Q}(S) \neq \varnothing$ such that $x_{i j} \in X$. From Table 2, we obtain:

$$
\begin{aligned}
\mathcal{Q}(S)= & \left\{\phi\left(x_{21}\right), \phi\left(x_{23}\right), \phi\left(x_{24}\right), \phi\left(x_{32}\right),\right. \\
& \left.\phi\left(x_{34}\right), \phi\left(x_{42}\right), \phi\left(x_{43}\right)\right\} \\
= & \{(170,170,170),(224,208,187), \\
& (103,183,255),(205,205,216), \\
& (202,210,187),(121,212,211)\}
\end{aligned}
$$

Hence we get

$$
\Phi^{*} S=\left\{x_{21}, x_{22}, x_{23}, x_{24}, x_{32}, x_{33}, x_{34}, x_{42}, x_{43}\right\}
$$

as shown in Fig. 4.

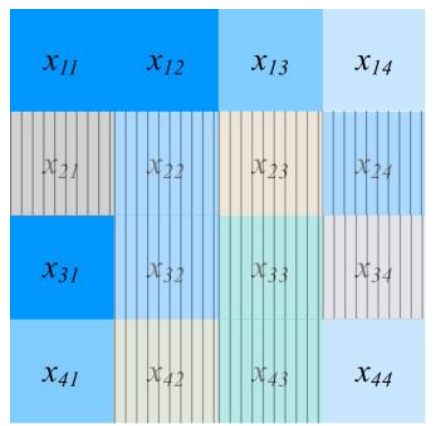

Figure 4: $\Phi^{*} S$

by Definition 3.10., since

1. For all $x_{i j}, x_{k l} \in S, x_{i j} \cdot x_{k l} \in \Phi^{*} S$,

2. For all $x_{i j}, x_{k l}, x_{m n} \in A,\left(x_{i j} \cdot x_{k l}\right) \cdot x_{m n}=$ $x_{i j} \cdot\left(x_{k l} \cdot x_{m n}\right)$ property holds in $\Phi^{*} S$,

are satisfied, the subimage $S$ of the digital image $X$ is indeed an approximately semigroup in descriptive proximity space $\left(X, \delta_{\Phi}\right)$ with binary operation ".". Moreover, since $x_{i j} \cdot x_{k l}=x_{k l}$. $x_{i j}$, for all $x_{i j}, x_{k l} \in S$ property holds in $\Phi^{*} S, S$ is a commutative approximately semigroup.

Definition 3.13. Let $T$ be a nonempty subset of approximately semigroup $S$ in $\left(X, \mathcal{R}_{\delta_{\phi}}\right)$. $T$ is called an approximately subsemigroup of $S$ if $T T \subseteq \Phi^{*} T$. In other words, $T$ is an approximately semigroup with the binary operation of $S$ restricted to $T$.

Example 3.14.From Example 3.12., let we consider approximately semigroup $S=\left\{x_{21}, x_{23}, x_{24}, x_{32}, x_{34}, x_{42}, x_{43}\right\}$ in descriptive proximity space $\left(X, \delta_{\Phi}\right)$ with binary operation ". ".

Let $T=\left\{x_{21}, x_{23}, x_{32}\right\}$ be a subimage (subset) of $S \subset X$. We can compute the descriptively upper approximation of $T$ by using the Definition 3.2. $\Phi^{*} T=\left\{x_{i j} \in X: x_{i j} \delta_{\phi} T\right\}, \quad$ where $\mathcal{Q}(T)=$ $\left\{\phi\left(x_{i j}\right): x_{i j} \in T\right\}$. Then $\phi\left(x_{i j}\right) \cap \mathcal{Q}(T) \neq \varnothing$ such that $x_{i j} \in X$. By Table 2, we obtain

$$
\begin{aligned}
\mathcal{Q}(T)= & \left\{\phi\left(x_{21}\right), \phi\left(x_{23}\right), \phi\left(x_{32}\right)\right\} \\
= & \{(170,170,170),(224,208,187), \\
& (103,183,255)\} .
\end{aligned}
$$

Then we get $\Phi^{*} T=\left\{x_{21}, x_{22}, x_{23}, x_{32}\right\}$. By Definition 3.13., since $T T \subseteq \Phi^{*} T$ property holds, the subimage $T$ of the digital image $S \subset X$ is indeed an approximately subsemigroup in descriptive proximity space $\left(X, \delta_{\Phi}\right)$ with binary operation ".".

Definition 3.15. Let $\left(X, \mathcal{R}_{\delta_{\Phi}}\right)$ be descriptive proximal relator space, $S$ be a approximately semigroup and $\emptyset \neq I \subseteq S$. 
(1) $I$ is called an approximately left ideal of $S$ if $\Phi^{*} I$ be a left ideal of $S$, that is $S\left(\Phi^{*} I\right) \subseteq \Phi^{*} I$.

(2) $I$ is called an approximately right ideal of $S$ if $\Phi^{*} I$ is a right ideal of $S$, that is $\left(\Phi^{*} I\right) S \subseteq \Phi^{*} I$.

(3) $I$ is called an approximately bi-ideal of $S$ if $\Phi^{*} I$ is a bi-ideal of $S$, that is $\left(\Phi^{*} I\right) S\left(\Phi^{*} I\right) \subseteq \Phi^{*} I$.

Example 3.16. From Example 3.12., let we consider approximately semigroup $S=\left\{x_{21}, x_{23}, x_{24}, x_{32}, x_{34}, x_{42}, x_{43}\right\}$ in descriptive proximity space $\left(X, \delta_{\Phi}\right)$ with binary operation ". ".

Let $I=\left\{x_{21}, x_{23}, x_{24}, x_{32}, x_{42}\right\}$ be a subimage (subset) of $S \subset X$. We can compute the descriptively upper approximation of $I$ by Definition 3.2. $\Phi^{*} I=\left\{x_{i j} \in X: x_{i j} \delta_{\phi} I\right\}$, where $\mathcal{Q}(I)=\left\{\phi\left(x_{i j}\right): x_{i j} \in I\right\}$. Then $\phi\left(x_{i j}\right) \cap \mathcal{Q}(I) \neq \varnothing$ such that $x_{i j} \in X$. From Table 2, we obtain

$$
\begin{aligned}
\mathcal{Q}(I)= & \left\{\phi\left(x_{21}\right), \phi\left(x_{23}\right), \phi\left(x_{24}\right), \phi\left(x_{32}\right), \phi\left(x_{42}\right)\right\} \\
= & \{(170,170,170),(224,208,187), \\
& (103,183,255),(202,210,187)\}
\end{aligned}
$$

Then we get $\Phi^{*} I=\left\{x_{21}, x_{22}, x_{23}, x_{24}, x_{32}, x_{42}\right\}$. By Definition 3.15., since $S\left(\Phi^{*} I\right) \subseteq \Phi^{*} I$ property holds, the subimage $I$ is indeed an approximately left ideal of the digital image $S$ in descriptive proximity space $\left(X, \delta_{\Phi}\right)$ with binary operation ".". Furthermore, since $S$ is a commutative approximately semigroup, we observe that $I$ is also approximately right and bi-ideal of $S$.

Example 3.17.By Examples 3.12. and 3.14., let we consider approximately subsemigroup $T=$ $\left\{x_{21}, x_{23}, x_{32}\right\} \subset S$ in descriptive proximity space $\left(X, \delta_{\Phi}\right)$ with binary operation ". ".

By Example 3.14. we know that descriptively upper approximation of $T$ is $\Phi^{*} T=\left\{x_{21}, x_{22}, x_{23}, x_{32}\right\}$. Then by Definition 3.15., since $S\left(\Phi^{*} T\right) \subseteq \Phi^{*} T$ property holds, the approximately subsemigroup (subimage) $T$ is indeed an approximately left ideal of the digital image $S$ in descriptive proximity space $\left(X, \delta_{\Phi}\right)$ with binary operation " . ". Furthermore, since $S$ is a commutative approximately semigroup, we observe that $T$ is also approximately right and bi-ideal of $S$.
In Example 3.17., we observe that the approximately subsemigroup (subimage) $T$ is indeed an approximately left ideal of $S$ in $\left(X, \delta_{\Phi}\right)$.

Example 3.18. From Example 3.12., let we consider approximately semigroup $S=\left\{x_{21}, x_{23}, x_{24}, x_{32}, x_{34}, x_{42}, x_{43}\right\}$ in descriptive proximity space $\left(X, \delta_{\Phi}\right)$ with binary operation ". ".

Let $K=\left\{x_{34}, x_{43}\right\}$ be a subimage (subset) of $S \subset$ $X$. We can compute the descriptively upper approximation of $K$ by using the Definition 3.2. $\Phi^{*} K=\left\{x_{i j} \in X: x_{i j} \delta_{\phi} K\right\}$, where $\mathcal{Q}(K)=$ $\left\{\phi\left(x_{i j}\right): x_{i j} \in K\right\}$. Then $\phi\left(x_{i j}\right) \cap \mathcal{Q}(K) \neq \varnothing$ such that $x_{i j} \in X$. From Table 2, we obtain

$$
\begin{aligned}
\mathcal{Q}(K) & =\left\{\phi\left(x_{34}\right), \phi\left(x_{43}\right)\right\} \\
& =\{(205,205,216),(121,212,211)\}
\end{aligned}
$$

Then we get $\Phi^{*} K=\left\{x_{34}, x_{33}, x_{43}\right\}$. By Definition 3.13., since $K K \subseteq \Phi^{*} K$ property holds, the subimage $K$ of the digital image $S \subset X$ is indeed an approximately subsemigroup in $\left(X, \delta_{\Phi}\right)$ with binary operation ". ". But since

$$
S\left(\Phi^{*} K\right)\left\{x_{21}, x_{22}, x_{23}, x_{24}, x_{32}, x_{33}, x_{34}, x_{42}, x_{43}\right\} \sqsubset \Phi^{*} K
$$

subimage $K$ is not an approximately left ideal (right or bi-ideal) of $S$ in $\left(X, \delta_{\Phi}\right)$.

In addition, although a subimage (subset) $J=\left\{x_{24}, x_{42}\right\}$ of $S \subset X$ is also an approximately subsemigroup of $S$, it is not an approximately left ideal (right or bi-ideal) of $S$ in $\left(X, \delta_{\Phi}\right)$.

Theorem 3.19Let $\left(X, \mathcal{R}_{\delta_{\Phi}}\right)$ be descriptive proximal relator space and $S \subseteq X$.

(1) If $S$ is a semigroup in $X$, then $S$ is an approximately semigroup in descriptive proximal relator space.

(2) If $I$ is a left (right, bi) ideal of approximately semigroup $S$, then $I$ is an approximately left (right, bi) ideal of $S$.

Proof. (1) Suppose that $S \subseteq X$ be a semigroup. From Theorem 3.5.(1), $\emptyset \neq S \subseteq \Phi^{*} S$. Hence $x \cdot y \in \Phi^{*} S, \forall x, y \in S$ and $(x \cdot y) \cdot z=x \cdot(y \cdot z)$ property holds in $\Phi^{*} S, \forall x, y, z \in S$. Then $S$ is an 
approximately semigroup in descriptive proximal relator space.

(2) Suppose that $I$ be a left ideal of approximately semigroup $S$, that is $S I \subseteq I$. We know that $S \subseteq$ $\Phi^{*} S$. Hence, by Theorems 3.5.(5) and 3.8.,

$$
S\left(\Phi^{*} I\right) \subseteq\left(\Phi^{*} S\right)\left(\Phi^{*} I\right)=\Phi^{*}(S I) \subseteq \Phi^{*} I .
$$

As a result $\Phi^{*} I$ is a left ideal of approximately semigroupS, and so $I$ is an approximately left ideal of $S$. We can easily prove that $I$ is a approximately right ideal of $S$. Therefore, $I$ is an approximately left, right or bi-ideal of $S$.

The Theorem 3.19. prove that the concept of approximately semigroup (left, right or bi-ideal) is a generalized concept of a semigroup (left, right or bi-ideal).

Theorem 3.20.Let $\left(X, \mathcal{R}_{\delta_{\Phi}}\right)$ be descriptive proximal relator space and $S \subseteq X$ be a semigroup and $A \subseteq$ $S$.

(1) If $A$ is a subsemigroup of $S$, then $\emptyset \neq \Phi_{*} A$ is a subsemigroup of $S$.

(2) If $I$ is a left (right, bi) ideal of $S$, then $\emptyset \neq \Phi_{*} I$ is a left (right, bi) ideal of $\Phi_{*} S$.

Proof. (1) Suppose that $A$ be a subsemigroup of $S$, then by Theorems 3.9. and 3.5.(4),

$$
\left(\Phi_{*} A\right)\left(\Phi_{*} A\right) \subseteq \Phi_{*}(A A) \subseteq \Phi_{*} A .
$$

Consequently $\emptyset \neq \Phi_{*} A$ is a subsemigroup of $S \subseteq$ $X$.

(2) Suppose that $I$ is a left ideal of $S$, that is $S I \subseteq I$. Then, by Theorems 3.9. and 3.5.(4),

$$
\left(\Phi_{*} S\right)\left(\Phi_{*} I\right) \subseteq \Phi_{*}(S I) \subseteq \Phi_{*} I .
$$

As a result $\emptyset \neq \Phi_{*} I$ is a left ideal of $\Phi_{*} S$. The proofs of other cases can be written in a similar processes.

Theorem 3.21.Let $\left(X, \mathcal{R}_{\delta_{\Phi}}\right)$ be descriptive proximal relator space and $S \subseteq X$. If $I$ is a bi-ideal of $S$, then it is an approximately bi-ideal of $S$.
Proof. Suppose that $I$ is a bi-ideal of $S$. Then, by Theorems 3.8. and 3.5.(5),

$$
\begin{aligned}
\left(\Phi^{*} I\right)(S)\left(\Phi^{*} I\right) & \subseteq\left(\Phi^{*} I\right)\left(\Phi^{*} S\right)\left(\Phi^{*} I\right) \subseteq \Phi^{*}(I S I) \\
& \subseteq \Phi^{*} I .
\end{aligned}
$$

As a result, by Theorem 3.20.(2), $\Phi^{*} I$ is a bi-ideal of $S$, that is, $I$ is an approximately bi-ideal of $S$.

Theorem 3.22.Let $\left(X, \mathcal{R}_{\delta_{\Phi}}\right)$ be descriptive proximal relator space and $S \subseteq X$. If $I$ is a bi-ideal of $S$, then $\emptyset \neq \Phi_{*} I$ is a bi-ideal of $\Phi_{*} S$.

Proof. Suppose that $I$ is a bi-ideal of $S$. Then, by Theorems 3.9 and 3.5.(6),

$$
\left(\Phi_{*} I\right)\left(\Phi_{*} S\right)\left(\Phi_{*} I\right) \subseteq \Phi_{*}(I S I) \subseteq \Phi_{*} I .
$$

Consequently, by Theorem 3.20.(2), $\varnothing \neq \Phi_{*} I$ is a bi-ideal of $\Phi_{*} S$.

Theorem 3.23.Let $\left(X, \mathcal{R}_{\delta_{\Phi}}\right)$ be descriptive proximal relator space and $S \subseteq X$. If I and J are right and left ideals of $S$, respectively, then

$$
\Phi^{*}(I J) \subseteq\left(\Phi^{*} I\right) \cap\left(\Phi^{*} J\right) .
$$

Proof. Suppose that $I$ and $J$ are right and left ideals of $S$, respectively. Then $I J \subseteq I S \subseteq I$ and $I J \subseteq S J \subseteq J$. Thus $I J \subseteq I \cap J$. As a result, by Theorem 3.5.(5) and (7),

$$
\Phi^{*}(I J) \subseteq \Phi^{*}(I \cap J) \subseteq\left(\Phi^{*} I\right) \cap\left(\Phi^{*} J\right) .
$$

Theorem 3.24.Let $\left(X, \mathcal{R}_{\delta_{\Phi}}\right)$ be descriptive proximal relator space and $S \subseteq X$. If I and J are right and left ideals of $S$, respectively, then

$$
\Phi_{*}(I J) \subseteq\left(\Phi_{*} I\right) \cap\left(\Phi_{*} J\right) .
$$

Proof. Suppose that $I$ and $J$ are right and left ideals of $S$, respectively. Then $I J \subseteq I S \subseteq I$ and $I J \subseteq S J \subseteq J$. Thus $I J \subseteq I \cap J$. Consequently, by Theorem 3.5.(3) and (4),

$$
\Phi_{*}(I J) \subseteq \Phi_{*}(I \cap J) \subseteq\left(\Phi_{*} I\right) \cap\left(\Phi_{*} J\right) .
$$




\section{Acknowledgement}

This research has been financially supported by the Scientific Research Fund of Adıyaman University under grant no. FBEBAP2015/0009.

\section{References}

Clifford A. and Preston G., 1964. The Algebraic Theory of Semigroups, American Mathematical Society, Providence, R.I., xv+224pp.

Efremovic V.A., 1951. Infinitesimal spaces, Doklady Akad. Nauk SSSR (N.S.), 76, 341-343.

Efremovic V.A., 1952. The geometry of proximity I, Mat. Sb. (N.S.), 31(73) (1), 189-200.

Kovăr M., 2011. A new causal topology and why the universe is co-compact, arXive:1112.0817[math-ph] 1-15arXiv:1112.0817.

Lodato M., 1962. On topologically induced generalized proximity relations, Ph.D. thesis, Rutgers University.

Naimpally S.A. and Peters J.F., 2013. Topology with Applications, Topological Spaces via Near and Far. World Scientific, Singapore.

Peters J.F., 2013. Near sets: An introduction, Math. in Comp. Sci., 7 (1), 3-9.

Peters J.F. and Naimpally S.A., 2012. Applications of near sets.,Notices Amer. Math. Soc., 59 (4), 536-542.

Peters J.F., 2016. Proximal relator spaces,Filomat,30 (2), 469-472.

Peters J.F, İnan E. and Öztürk M.A., 2015. Exactness of Proximal Groupoid Homomorphisms, Adıyaman University Journal of Science, 5 (1), 1-13.

Peters J.F., İnan E. and Öztürk M.A., 2014. Spatial and descriptive isometries in proximity spaces, General Mathematics Notes, 21 (2), 1-10.

Szaz A., 1987. Basic tools and mild continuities in relator spaces, Acta Math. Hungar., 50, 177-201. 